OPEN ACCESS

Edited by:

Thomas Fath,

Macquarie University, Australia

Reviewed by:

Gabriele Loers,

Universitätsklinikum

Hamburg-Eppendorf, Germany Lidija Radenovic,

University of Belgrade, Serbia

*Correspondence:

Patricia F. Maness

srclab@med.unc.edu

Specialty section:

This article was submitted to

Cell Adhesion and Migration,

a section of the journal

Frontiers in Cell and Developmental

Biology

Received: 31 October 2018

Accepted: 16 January 2019

Published: 31 January 2019

Citation:

Mohan V, Gomez JR and

Maness PF (2019) Expression

and Function of Neuron-Glia-Related

Cell Adhesion Molecule (NrCAM)

in the Amygdalar Pathway.

Front. Cell Dev. Biol. 7:9.

doi: 10.3389/fcell.2019.00009

\section{Expression and Function of Neuron-Glia-Related Cell Adhesion Molecule (NrCAM) in the Amygdalar Pathway}

\author{
Vishwa Mohan, Julia R. Gomez and Patricia F. Maness* \\ Department of Biochemistry and Biophysics, University of North Carolina School of Medicine, Chapel Hill, NC, United States
}

Neuron-Glia related cell adhesion molecule (NrCAM) is a candidate autism risk factor that promotes axon guidance through cytoskeletal linkages in developing brain but its role in limbic circuitry has not been investigated. In situ hybridization (ISH) and immunofluorescence staining showed that NrCAM is expressed in the developing amygdalar pathway of mouse embryos during outgrowth of projections in the stria terminalis, a major limbic tract that interconnects the central amygdala $(\mathrm{CeA})$ with key targets in the bed nucleus of the stria terminalis (BNST). Analysis of fiber tracts in NrCAM mutant mice by Neurofilament protein immunohistochemistry showed pronounced defasciculation and misprojection of fibers in the ST. The defasciculation phenotype may result from impairment in NrCAM homophilic inter-axonal adhesion or axon repulsion from the secreted ligand Semaphorin 3F, which is expressed in limbic areas in proximity to the ST. Behavioral testing indicated that NrCAM null mice were impaired in contextdependent fear conditioning, in accord with altered amygdala-BNST connectivity, but displayed normal cued (tone-shock) conditioning. Results are consistent with the novel finding that NrCAM mediates fasciculation of axon fibers in the ST important for proper amygdalar-BNST circuitry and response to contextual fear conditioning.

Keywords: stria terminalis, NrCAM, bed nucleus of the stria terminalis, fear conditioning, limbic system

\section{INTRODUCTION}

During brain development a diversity of guidance cues and receptors is required for correct synaptic targeting of axons. One important group of guidance receptors is represented by immunoglobulin-class (Ig) cell adhesion molecules of the L1 family (L1-CAMs) (Sytnyk et al., 2017). Among these, Neuron-Glia related cell adhesion molecule (NrCAM), L1, and Close Homolog of L1 promote axon growth through trans homophilic binding of their extracellular domains and intracellular coupling of their conserved cytoplasmic domains to the actin cytoskeleton. Cytoskeletal coupling of L1-CAMs is achieved through direct binding to the actin adaptors Ankyrin and Ezrin-Radixin-Moesin proteins (ERM), as well as to PDZ-containing scaffold proteins PSD-95 and SAP102 (Buhusi et al., 2008; Schlatter et al., 2008; Demyanenko et al., 2014; Leshchyns'ka and Sytnyk, 2016). NrCAM is notable as a potential target for mutation in neurodevelopmental disease, as polymorphisms in the $\mathrm{NrCAM}$ locus have been associated with autism spectrum disorders (ASD) (Pinto et al., 2010; Voineagu et al., 2011; Sakurai, 2012). 
Moreover, male NrCAM knockout mice exhibit autism-related behaviors, including impaired sociability, cognitive rigidity, and repetitive behavior (Moy et al., 2009a).

Neuron-Glia related cell adhesion molecule mediates axon repulsion in response to the repellent ligand Semaphorin $3 \mathrm{~F}$ (Sema3F) (Falk et al., 2005; Demyanenko et al., 2011). In this role NrCAM functions as an integral component of the Sema3F holoreceptor complex, which comprises the co-receptor Neuropilin-2 (Npn2) and signaling subunit PlexinA3 (PlexA3) (Figure 1A). In the presence of Sema3F, NrCAM induces clustering of Npn2 and PlexA3 in the neuronal membrane to activate intrinsic PlexA3 Rap-GAP activity (Mohan et al., 2018). Sema3F-induced axon repulsion through NrCAM is a key mechanism for regulating guidance of thalamocortical (Demyanenko et al., 2011) and commissural axon projections (Falk et al., 2005). In the developing limbic system Sema3F and Npn2 are expressed at discrete locations, where they are required for proper development of the stria terminalis (ST), a nerve fiber bundle that interconnects the central amygdala (CeA) and bed nucleus of the ST (BNST) (Sahay et al., 2003). Connectivity between the CeA and BNST is critical for fear and stress responses, as well as for social interactions (Davis et al., 2010; Coria-Avila et al., 2014; Daniel and Rainnie, 2016; Lebow and Chen, 2016). Because a role for NrCAM in this limbic pathway has not been investigated, we hypothesized that NrCAM may be involved in regulating the development of ST projections between the CeA and BNST, and this might affect behavioral responses to contextual fear conditioning, which depends in part on amygdalar-BNST circuitry (Stamatakis et al., 2014).

To probe this hypothesis, we analyzed NrCAM expression in the amygdalar-BNST projection and studied the effects of NrCAM deletion on the structure and function of this limbic connection in NrCAM null mice. We found that NrCAM was expressed in the ST, CeA, and BNST during establishment of amygdalar connectivity, and that deletion of $\mathrm{NrCAM}$ in null mutant mice caused pronounced disruption of axonal tracts in the ST due to defasciculation of axon bundles. Behavioral testing showed further that NrCAM null mice were impaired for contextual fear conditioning. These novel results suggested that NrCAM mediates fasciculation of axons in the ST through homophilic adhesion or repellent guidance, important for contextual fear conditioning responses.

\section{MATERIALS AND METHODS}

\section{Mice}

Wild type (WT) and homozygous null NrCAM mutant mice were bred for over 10 generations to establish them on a C57BL/6 genetic background. Mice were group-housed in ventilated microisolator cages with free access to water and Prolab RMH 3000 chow. The housing room had a 12-h light/dark cycle (lights off at 7:00 p.m.). Embryonic day E0.5 was defined as the plug date and the day of birth as P0. All animal studies were approved by the Institutional Animal Care and Use Committee (IACUC) of The University of North Carolina School of Medicine at Chapel Hill (IACUC Protocol \# 15-114). Mice were handled according to the University of North Carolina IACUC policies and in accordance with NIH guidelines for humane care and use of laboratory animals. All the experiments were performed with male mice except experiments with mouse embryos, which were of mixed gender.

\section{NrCAM and Neurofilament Immunohistochemistry and Immunofluorescence Staining}

Wild type and NrCAM null littermates were anesthetized and perfused transcardially with $4 \%$ paraformaldehyde (PFA) buffered in phosphate buffered saline (PBS). For embryos, the head was subjected to fixation without perfusion, and cryo-protected by immersing in 30\% sucrose in PBS. Coronal cryostat sections $(24 \mu \mathrm{m})$ were mounted on slides and stored at $-80^{\circ} \mathrm{C}$. For immunoperoxidase staining, sections were subjected to antigen retrieval by brief exposure to $10 \mathrm{mM}$ citrate $\left(\mathrm{pH}\right.$ 6) at $100^{\circ} \mathrm{C}$. After three washes with PBS, endogenous peroxidase activity was quenched by treating $5 \mathrm{~min}$ with $3 \% \mathrm{H}_{2} \mathrm{O}_{2}$. After washing with $\mathrm{PBS}$, sections were blocked at room temperature (RT) in PBS containing 5\% goat serum and $0.3 \%$ Triton X-100. Sections were incubated overnight with monoclonal Neurofilament antibody 2H3 (DSHB, Iowa City, IA, United States) (1:200). For NrCAM sections were incubated with rabbit polyclonal antibody (AbCAM \#24344). We and others have used this antibody previously and validated the specificity of this antibody by immunostaining and immunoblotting on WT and NrCAM null samples (Amor et al., 2014; Demyanenko et al., 2014; Morelli et al., 2017; Mohan et al., 2018). Sections were washed with PBS and incubated with biotinylated antimouse secondary antibody (1:200, Vector Laboratories) for $1 \mathrm{~h}$ at RT. Immunoreactivity was visualized using a Vectastain avidinbiotinylated peroxidase kit. Sections were dehydrated in an ascending series of 50,70, 90, and 100\% ethanol and cleared in xylene before mounting in Permount (Fisher Chemicals). Bright-field images were taken on a Zeiss Axioplan microscope. For quantitative estimation of the degree of fasciculation of the ST, images of Neurofilament-stained coronal sections $(24 \mu \mathrm{m}$; 3 sections per brain, spanning the entire ST) of WT and NrCAM null mutant brains (P30; 3 mice per genotype) were analyzed at the same magnification and rostro-caudal level using Image J. The line measuring tool was used to obtain the width of the ST at multiple locations along its length, and the average width calculated. The average width of ST in all sections was determined for each mouse, and the mean width per genotype (+SEM) was calculated. Statistically significant differences in the mean width of the ST were analyzed by the $t$-test (two tailed, unequal variances; $\left.{ }^{*} p<0.05\right)$.

For double immunofluorescence staining, sections were incubated overnight with Neurofilament antibody 2H3 (1:400) and NrCAM antibody (AbCAM \#24344; 1:200). After washing in PBS, sections were treated with anti-mouse AlexaFluor 488 and anti-rabbit AlexaFluor 555 secondary antibodies (1:500, LifeTechnologies) for $1 \mathrm{~h}$ at RT. After washing in PBS, sections were mounted on slides using ProLong gold antifade mountant (Invitrogen). Images were obtained using a Zeiss LSM700 
A



B

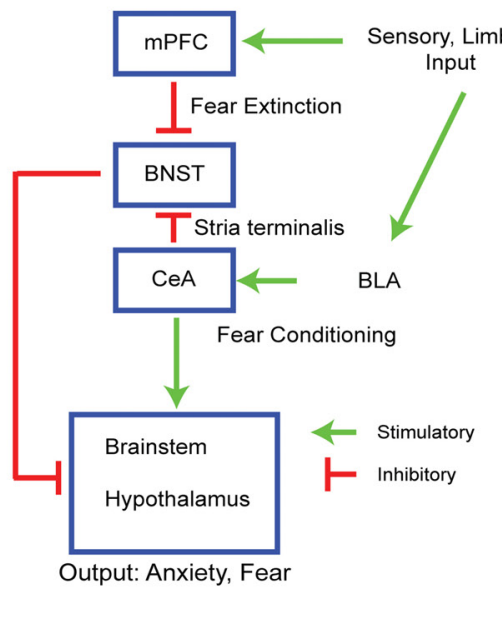

C



E



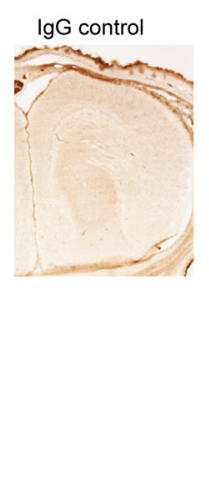

$\mathbf{F}$

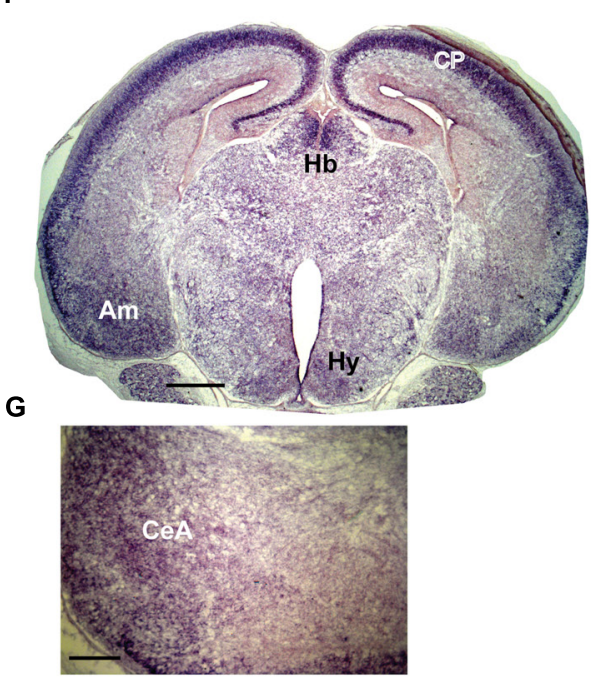

$\mathrm{H}$

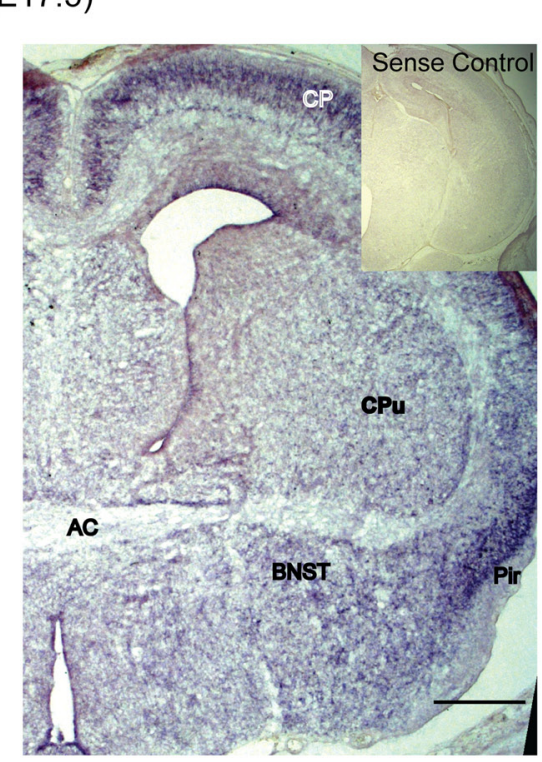

FIGURE 1 | Continued 
FIGURE 1 | NrCAM expression in the amygdalar pathway. (A) Molecular model of Sema3F holoreceptor complex. NrCAM interacts with the Sema3F co-receptor Npn-2, which binds PlexinA3 with Rap-GTPase activating protein (Rap-GAP) activity, leading to axon repulsion. The NrCAM cytoplasmic domain recruits actin cytoskeletal adapters Ezrin-Radixin-Moesin (ERM), Ankyrin, and PDZ-interacting scaffold protein SAP102. (B) Schematic of cortico-limbic connectivity. Sensory and limbic input is received by the basolateral amygdala (BLA). The BLA promotes fear conditioning by connections with the central amygdala (CeA). The CeA sends inhibitory GABAergic projections to the BNST and stimulates the brainstem and hypothalamus. Input to the mPFC promotes fear extinction by inhibiting the BNST. (C) Immunohistochemical staining (IHC) for NrCAM in coronal brain sections from E17.5 WT mouse brain. NrCAM immunoperoxidase staining is observed in developing nuclei (CeA, BLA, basomedial) of the amygdala, cortex, and thalamus. Scale bar = $1000 \mu \mathrm{m}$. (D) NrCAM immunoreactivity in E17.5 mouse brain is present in the BNST, cortex, anterior commissure (AC), and caudate putamen (CPu). Scale bar = $1000 \mu \mathrm{m}$. (E) Control labeling with nonimmune IgG. (F) In situ hybridization (ISH) of NrCAM mRNA in coronal sections of E17.5 mouse brain shows enrichment in the amygdala (Am), cortical plate (CP), habenula (Hb), and hypothalamus $(\mathrm{Hy})$, Scale bar $=1000 \mu \mathrm{m}$. (G) Higher magnification showing ISH of NrCAM mRNA in the region of the CeA nucleus of the amygdala at E17.5. Scale bar $=250 \mu \mathrm{m}$. (H) ISH of NrCAM mRNA in E17.5 mouse brain also shows expression in the BNST adjacent to the anterior commissure (AC), as well as the cortical plate (CP) and piriform cortex (Pir). Scale bar $=1000 \mu \mathrm{m}$.

confocal microscope at identical settings to compare WT and mutant staining.

\section{In situ Hybridization of NrCAM Transcripts}

A pBlueScript (pBS) plasmid containing mouse NrCAM cDNA (GenBank accession number AJ54321, nucleotides 460-1470) was used to generate digoxigenin (DIG)-labeled riboprobes for in situ hybridization (ISH) to NrCAM transcripts as described (Demyanenko et al., 2011) in the histology core facility of the UNC Neuroscience Research Center. Briefly, fetal mouse brains (E17.5) were fixed in 4\% PFA buffered in PBS overnight at $4^{\circ} \mathrm{C}$. Brains were cryo-protected by immersing in an ascending series of 10,20 , and $30 \%$ sucrose solution in PBS at $4^{\circ} \mathrm{C}$. Coronal sections were cut at $20 \mu \mathrm{m}$ on a cryostat and mounted onto slides. ISH was performed using digoxygenin-labeled anti-sense and control sense riboprobes as reported (Bartsch et al., 1994; Colbert et al., 1995), and images were captured digitally on a Zeiss Axioplan 2 microscope.

\section{Behavioral Testing}

Adult WT and NrCAM homozygous null littermates (14 WT $\mathrm{NrCAM}^{+/+}$and $20 \mathrm{NrCAM}^{-/-}$male mice) were evaluated for fear memory in cued-and contextual fear conditioning tests using the Near-Infrared image tracking system (Med Associates Inc., Burlington, VT, United States) in the UNC Mouse Behavioral Phenotyping Core of the Carolina Institute for Developmental Disabilities (Dr. Sheryl S. Moy, Director). This apparatus has a sound attenuating cubicle with a near infrared/visible lighting system, which has been described and used extensively for fear conditioning in mice and other species (Huang et al., 2013; Zhu et al., 2014). The fear conditioning chamber has a digital video camera and infrared sensors on each side that permit freezing to be recorded by measuring the latency to break infrared beams. Levels of freezing (no movement for $0.5 \mathrm{~s}$ ) were automatically measured by the image tracking software. The procedure was conducted across 3 days. On the first day, mice were given a 7 -min training session. Mice were placed in the test chamber, contained in a sound-attenuating box, and allowed to explore for $2 \mathrm{~min}$. The mice were then exposed to a 30 -s tone $(80 \mathrm{~dB})$, followed by a 2 -s scrambled foot shock (0.4 mA). Mice received two additional shock-tone pairings, with $80 \mathrm{~s}$ between each pairing. Context-dependent learning was evaluated on the second day of testing. Mice were placed back into the original test chamber, and levels of freezing (immobility) were determined across a 5-min session. On the third day of testing, mice were evaluated for associative learning to the auditory cue in a final 5-min session. The fear conditioning test chamber was cleaned thoroughly with $70 \%$ ethanol before each session. After conditioning, animals were returned to home cages in standard housing. The conditioning chambers were modified using a Plexiglas insert to change the wall and floor surface, and a novel odor (vanilla flavoring) was added to the sound-attenuating box. Mice were placed in the modified chamber and allowed to explore. After $2 \mathrm{~min}$, the acoustic stimulus was presented for a 3-min period. Levels of freezing before and during the stimulus were obtained by the image tracking system. All mice were 6-8 months of age at the time of behavioral testing. A small subset of these mice (3 WT and $5 \mathrm{NrCAM}-$ /-) were tested 6 months previously in a study on oxytocin effects in a social approach task, and were not handled in the interim. No effect of oxytocin on sociability was observed in any of these mice, therefore, it is unlikely that this could affect the observations reported here. Results were analyzed using repeated measures analysis of variance (ANOVAs) with the factor genotype (WT or NrCAM null). Fisher's protected least-significant difference (PLSD) tests was used for comparing group means only when a significant $\mathrm{F}$ value was determined in the overall ANOVA. For all comparisons, significance was set at $p<0.05$.

\section{RESULTS}

\section{NrCAM Is Expressed in Limbic Regions of the Embryonic Mouse Brain During Establishment of Amydgalar Connectivity}

In cortico-limbic circuitry (Figure 1B) sensory and limbic inputs to the basolateral amygdala promote conditioned fear through connectivity with the CeA ultimately targeting the brain stem and hypothalamus (Hy) to evoke autonomic responses of fear and anxiety. GABAergic neurons in the $\mathrm{CeA}$ are notable in making inhibitory connections with the BNST that dampen these responses (Knobloch et al., 2012; Kim et al., 2013). In addition, the medial prefrontal cortex (mPFC) provides "top-down" extinction of responses through 
connections with the BNST (Amano et al., 2010; John et al., 2013).

To investigate a potential involvement of $\mathrm{NrCAM}$ in influencing connectivity between the amygdala and BNST we analyzed NrCAM expression by immunohistochemistry using immunoperoxidase staining, and by ISH in WT mouse embryos at E17.5, when CeA to BNST projections extend and grow toward their target areas (Sahay et al., 2003). Immunoperoxidase staining for NrCAM protein showed widespread NrCAM immunoreactivity in the cortex, thalamus, and amygdalar area (Figure 1C). In the E17.5 amygdala NrCAM was expressed at sites corresponding to central (CeA), basolateral (BLA), and basomedial (BMA) amygdalar nuclei (Figure 1C). NrCAM staining was also seen in the BNST, caudate putamen $(\mathrm{CPu})$, and anterior commissure (AC) (Figure 1D). Control staining with nonimmune IgG was minimal (Figure 1E). NrCAM immunoreactivity marks the location of NrCAM in cell bodies as well as its presence in fibers traversing these regions. To identify the location of NrCAM expressing soma, NrCAM transcripts were localized by ISH in brain sections of WT mice at E17.5. Brain regions were identified by neuroanatomical landmarks and comparison to atlas coordinates for E17.5 mouse brain (Paxinos et al., 2007). NrCAM transcripts were detected by hybridization to an anti-sense riboprobe and found to be abundant in the cortical plate (CP) and amygdala (Am) (Figure 1F). Labeling was also present in the medial habenula $(\mathrm{Hb})$ and $\mathrm{Hy}$. When imaged at higher magnification, $\mathrm{NrCAM}$ transcripts were evident in the CeA (Figure 1G). In more rostral sections NrCAM transcripts were present in the BNST adjacent to the AC, as well as the $\mathrm{CPu}$ (Figure 1H). High levels of expression are observed in the $\mathrm{CP}$ and piriform cortex (Pir). Control hybridization to the sense riboprobe elicited no detectable labeling (Figure $\mathbf{1 H}$, inset). These results showed that NrCAM was expressed in the CeA-BNST pathway during axon targeting in the developing mouse limbic system.

\section{Axons in the Stria Terminalis Express NrCAM, and Are Defasciculated in NrCAM Null Mice}

To validate the specificity of the NrCAM antibody, double immunofluorescence staining for $\mathrm{NrCAM}$ and Neurofilament protein was performed at postnatal day P25 in WT and NrCAM null mutant neocortex, as this region is known to express high levels of NrCAM postnatally (Mohan et al., 2018) (Figures 2A,B). NrCAM immunofluorescence staining was prominent in WT mice as shown in the cortex, whereas it was absent in NrCAM null cortex (Figures 2A,B). Neurofilament immunofluorescence staining of axons was clearly evident in the cortex of both genotypes. To assess the localization of NrCAM in the ST postnatally, we performed immunofluorescence staining for NrCAM and Neurofilament in brain sections through the ST of WT mice at P25. NrCAM immunofluorescence staining was present in Neurofilament-positive fibers of the ST (Figure 2C). NrCAM staining was also evident in the triangular and lateral septum (LS). In contrast, NrCAM immunofluorescence was absent from the ST and adjacent areas in the brain of NrCAM null mice, validating the specificity of the staining (Figure 2D). ST fibers originating in the CeA extend toward the BNST at approximately E17.5 and complete synaptic targeting as development approaches P30 (Sahay et al., 2003). We also assessed the expression of $\mathrm{NrCAM}$ in the amygdalar region of WT mice at two key developmental stages, E17.5 and P25, by immunofluorescence staining. Results showed that $\mathrm{NrCAM}$ was more prominently expressed in embryos at E17.5 then declined to lower levels postnatally at P25 (Figure 2E).

The ST is a highly fasciculated axon tract in the mature limbic system (Stamatakis et al., 2014). The presence of NrCAM in the ST suggested that homophilic or heterophilic interactions within the extracellular region of NrCAM might play a role in fasciculation of axon bundles comprising the ST. To visualize the ST, we performed immunoperoxidase labeling of Neurofilament protein in mice at postnatal day P30, when the tract is fully formed. In WT mice the ST appeared as a highly fasciculated fiber bundle dorsal to the AC (Figure 2F, left panel). In sections of NrCAM null mutant mice matched for rostrocaudal level, the ST appeared broader in width, with fibers that strayed from the main tract and appeared disorganized (Figure $2 \mathbf{F}$, right panel). At higher magnification of WT brain sections from other mice, ST fibers ran as a relatively tight bundle, whereas in NrCAM null mice fibers appeared defasciculated (Figures 2G,H; arrows). To provide a quantitative estimate of defasciculation, the average width of the ST was measured in serial coronal sections matched for level from WT and NrCAM mutant brains ( $n=3$ mice/genotype), and compared for significant mean differences. This analysis indicated that the mean width of the ST in NrCAM mutant mice was significantly increased compared to WT $\left(t\right.$-test, $\left.{ }^{*} p<0.05\right)$ (Figure 2I).

\section{Contextual Fear Conditioning Is Impaired in NrCAM Null Mice}

Dysconnectivity between the CeA and BNST may disrupt limbic circuits involved in regulation of behavioral responses such as fear and sociability (Lebow and Chen, 2016). male NrCAM null mutant mice exhibit sociability deficits, cognitive inflexibility, and hyper-responsiveness to sensory stimuli but fear conditioning was not tested (Moy et al., 2009b).To determine if loss of NrCAM affected behavior responses involving limbic function, cued (tone-shock) and contextual fear conditioning were analyzed in adult WT and NrCAM null male mice. We analyzed male WT and NrCAM null mutant mice in fear conditioning, because only males showed impairments in sociability, reversal learning, and sensory gating (Moy et al., 2009b). Moreover, ASD is more common in males than females across age groups (Hull et al., 2017). We tested both cued and context-dependent fear conditioning in well-characterized learning paradigms (Huang et al., 2013; Zhu et al., 2014). Cued fear conditioning involves circuits in the CeA, basolateral amydgala, and periaqueductal gray of the brainstem, whereas contextual fear conditioning is mediated through circuitry involving the CeA and BNST, as well as the hippocampus and mPFC (Stamatakis et al., 2014). Deletion of NrCAM in NrCAM mutant mice led to selective deficits in context-dependent 



FIGURE 2 | NrCAM expression and effect of deletion on the ST of NrCAM Null Mice. (A,B) Representative images of immunofluorescence staining for NrCAM (red) and Neurofilament protein (green) in coronal sections through the brain of P25 WT and NrCAM null mice. Scale bar = $150 \mu \mathrm{m}$. (C) NrCAM immunofluorescence staining was prominently localized in Neurofilament-positive fibers of the ST in WT mice (P25). NrCAM staining was also present in the lateral and triangular septa (LS, TS). (D) NrCAM immunostaining was not detectable in the Neurofilament-positive ST or other regions in this region of the NrCAM null brain (P25). Scale bar $=100 \mu \mathrm{m}$. (E) NrCAM immunofluorescence staining in the central amygdalar nucleus (CeA) was prominent in WT embryos at E17.5 and declined to lower levels at P25. Scale bar $=100 \mu \mathrm{m}$. (F-H) Neurofilament immunoperoxidase staining in coronal sections of WT mouse brain at P30. (F) Left panel: WT brain showing the ST entering the BNST as a tightly fasciculated bundle. Right panel: NrCAM null brain showing the ST with defasciculated fibers (arrow). Abbreviations: BNST (LD-lateral dorsal and MV-medial ventral areas), anterior commissure (AC), caudate putamen (CPu). Scale bar $=1000 \mu \mathrm{m}$. (G) Images from different P30 individual mice (upper and lower panels) showing the ST as a well-formed bundle in WT brains, compared to NrCAM null brains, in which ST axons appear defasciculated (arrows). LS, lateral septum. Scale bar $=250 \mu \mathrm{m}$. (H) High magnification images showing defasciculated and straying ST fibers (arrows) in NrCAM null compared to WT mice at P30. Scale bar $=200 \mu \mathrm{m}$. (I) Histogram comparing the mean width of the ST in WT and NrCAM null mice (P30), measured in three sections spanning the ST per mouse, as described in Materials and Methods. Mean + SEM; $n=3$ mice/genotype; ${ }^{*} p<0.05$.

learning, measured by percent freezing over a 5 min period on day 2 following the training day 1 [genotype $x$ time interaction, $\left.F(5,105)=2.50,{ }^{*} p=0.0349\right]$ (Figure 3A). In contrast, both the
WT and mutant mice showed similar levels of cue-dependent learning measured on day 3 (Figure 3B). No group differences were observed in baseline levels of freezing (measured as percent 
A

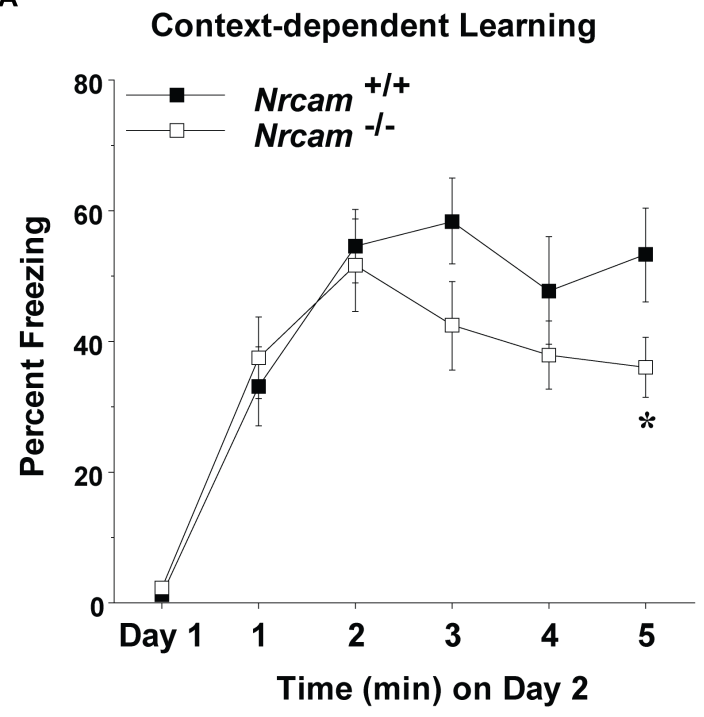

B



C



FIGURE 3 | Impaired contextual fear conditioning in NrCAM null mice. (A) Baseline levels of freezing behavior before shock exposure were determined on day 1 (the training day). Contextual learning was evaluated on day 2 of testing. (B) Cued learning was determined on day 3, using an 80-decibel acoustic stimulus in the last 3 min of the 5 -min session. Data shown represent means (+SEM), $\left.{ }^{*} p=0.0349\right)$. (C) Hypothetical model showing axonal projections of the ST between the CeA and BNST. Fasciculation of axons expressing NrCAM in the ST may be mediated by repulsion from Sema3F secreted by cells in the caudate putamen (CPu), or through homophilic NrCAM-NrCAM adhesion between axons.

time immobile) during the first exposure to the conditioned fear chamber.

\section{DISCUSSION}

In this study we analyzed the expression and function of NrCAM in promoting axon fasciculation in the ST, a key tract connecting the CeA with targets in the BNST. In embryonic mouse brain (E17.5) NrCAM was found to be expressed in developing amygdalar nuclei, including the CeA, during establishment of connectivity with the BNST. Deletion of
NrCAM in homozygous null mutant male mice revealed a phenotype of axon defasciculation in the ST, suggesting that NrCAM promotes axon bundling and proper targeting of these projections. Behavioral testing of WT and NrCAM null mice showed that NrCAM loss impaired contextual fear conditioning, a response dependent on CeA-BNST circuitry. These results demonstrate a novel function for NrCAM in development of limbic circuitry.

Neuron-glia-related cell adhesion molecule mediates repellent signaling in axons as an obligate component of the Sema3F holoreceptor complex. As shown in Figure 1A, NrCAM binds the Sema3F co-receptor Npn2 and promotes signaling through 
the associated PlexA3 subunit. It has been reported that Sema3F transcripts are expressed in the embryonic $\mathrm{CPu}$, which borders the ST, while Npn2 transcripts are present in the CeA and other amygdaloid nuclei (Sahay et al., 2003). Sema3F- and Npn2-deficient mice display disorganized and defasciculated axons of amygdalar projections to the BNST (Sahay et al., 2003), like the phenotype of NrCAM null mice observed here. Our results showed that $\mathrm{NrCAM}$ was localized to fibers in the developing ST (Figure 2C). Accordingly, loss of NrCAM may impair a repellent response of $\mathrm{CeA}$ axons to Sema3F, resulting in defasciculation and mistargeting of ST axons, as depicted in Figure 3C. In support of this notion, Sema3F deletion in mice has been associated with abnormalities in fear-related responses in mice (Matsuda et al., 2016). Homophilic adhesion between axons through NrCAM-NrCAM binding could also contribute to ST axon fasciculation. Both mechanisms likely depend on cytoskeletal interactions of the NrCAM cytoplasmic domain with Ankyrin, ERM, and/or PDZ-domain scaffold proteins.

The CeA to BNST projection is topographically specified, such that CeA axons project to anterolateral nuclei in the BNST, whereas medial amygdalar axons project to posterior and anteromedial BNST nuclei (Dong et al., 2001). A limitation of the present work is that axon tracing has not been performed to determine if topographic targeting relies on selective fasciculation of fibers in the ST through NrCAM interactions. Analogously, Sema3A, a closely related secreted Semaphorin, promotes selective fasciculation of olfactory sensory fibers in a pre-target sorting mechanism for olfactory topographic map formation in mice (Imai et al., 2009). Also, axons from the amygdala reach the BNST via two distinct pathways: the ST (dorsal) and ansa peduncularis (ventral) (Dong et al., 2001). The integrity of the ansa peduncularis has not been investigated for an effect of NrCAM deletion.

Lesion and pharmacological studies have indicated that the BNST is involved in the modulation of innate fear responses (Davis et al., 1997; Xu et al., 2012). The present finding that NrCAM null mice exhibit decreased contextual fear conditioning is in accord with altered CeA-BNST connectivity in modulating fear (Amano et al., 2010). In contrast, NrCAM deletion did not affect cued fear conditioning, which relies on connections between the $\mathrm{CeA}$ and basolateral amygdala

\section{REFERENCES}

Amano, T., Unal, C. T., and Pare, D. (2010). Synaptic correlates of fear extinction in the amygdala. Nat. Neurosci. 13, 489-494. doi: 10.1038/nn.2499

Amor, V., Feinberg, K., Eshed-Eisenbach, Y., Vainshtein, A., Frechter, S., Grumet, M., et al. (2014). Long-term maintenance of $\mathrm{Na}+$ channels at nodes of Ranvier depends on glial contact mediated by gliomedin and NrCAM. J. Neurosci. 34, 5089-5098. doi: 10.1523/JNEUROSCI.4752-13.2014

Bartsch, U., Faissner, A., Trotter, J., Dorries, U., Bartsch, S., Mohajeri, H., et al. (1994). Tenascin demarcates the boundary between the myelinated and nonmyelinated part of retinal ganglion cell axons in the developing and adult mouse. J. Neurosci. 14, 4756-4768. doi: 10.1523/JNEUROSCI.14-08-04756. 1994

Buhusi, M., Schlatter, M. C., Demyanenko, G. P., Thresher, R., and Maness, P. F. (2008). L1 interaction with ankyrin regulates mediolateral topography in the
(Stamatakis et al., 2014). Our studies differ in part from Matzel et al. (2008), who reported no effect of NrCAM deletion on cued or contextual fear conditioning using a water licking paradigm. In that study, NrCAM mutant mice were on a hybrid background of Sv129 and outbred Swiss Webster, whereas our mice were predominantly on C57/BL6. Finally, it should be noted that NrCAM loss may disrupt connectivity within other brain regions that contribute to contextual fear conditioning, such as the hippocampus and mPFC. In this regard, the increased dendritic spine density of mPFC pyramidal neurons in NrCAM mutant mice (Mohan et al., 2018) might impact "top-down" regulation of fear conditioning responses.

In summary, the present study demonstrates the novel finding that NrCAM is expressed in the embryonic amygdala-BNST pathway and is required for proper axon fasciculation of the ST. Mistargeting of axons in the CeA-BNST projection may contribute to impaired contextual fear conditioning in mice with loss-of-function mutations in NrCAM.

\section{AUTHOR CONTRIBUTIONS}

VM and JG performed NrCAM expression experiments and analysis of axon projections. Mouse behavioral testing was performed in the UNC Mouse Behavioral Phenotyping Core. PM designed and analyzed the experiments, and wrote the manuscript with VM.

\section{FUNDING}

This work was supported by National Institute of Health Grant R01-MH101605 (PM), and NIH Center grant to the UNC Carolina Institute for Developmental Disabilities U54 HD079124.

\section{ACKNOWLEDGMENTS}

Dr. Sheryl Moy (Director, UNC Mouse Behavioral Phenotyping Core) and Natallia Riddick are gratefully acknowledged for mouse behavioral testing.

retinocollicular projection. J. Neurosci. 28, 177-188. doi: 10.1523/JNEUROSCI. 3573-07.2008

Colbert, M. C., Rubin, W. W., Linney, E., and Lamantia, A. S. (1995). Retinoid signaling and the generation of regional and cellular diversity in the embryonic mouse spinal cord. Dev. Dyn. 204, 1-12. doi: 10.1002/aja.1002040102

Coria-Avila, G. A., Manzo, J., Garcia, L. I., Carrillo, P., Miquel, M., and Pfaus, J. G. (2014). Neurobiology of social attachments. Neurosci. Biobehav. Rev. 43, 173-182. doi: 10.1016/j.neubiorev.2014.04.004

Daniel, S. E., and Rainnie, D. G. (2016). Stress modulation of opposing circuits in the bed nucleus of the stria terminalis. Neuropsychopharmacology 41, 103-125. doi: 10.1038/npp.2015.178

Davis, M., Walker, D. L., and Lee, Y. (1997). Amygdala and bed nucleus of the stria terminalis: differential roles in fear and anxiety measured with the acoustic startle reflex. Philos. Trans. R. Soc. Lond. B Biol. Sci. 352, 1675-1687. doi: 10.1098/rstb.1997.0149 
Davis, M., Walker, D. L., Miles, L., and Grillon, C. (2010). Phasic vs sustained fear in rats and humans: role of the extended amygdala in fear vs anxiety. Neuropsychopharmacology 35, 105-135. doi: 10.1038/npp.2009.109

Demyanenko, G. P., Mohan, V., Zhang, X., Brennaman, L. H., Dharbal, K. E., Tran, T. S., et al. (2014). Neural cell adhesion molecule NrCAM regulates semaphorin 3F-induced dendritic spine remodeling. J. Neurosci. 34, 11274-11287. doi: 10. 1523/JNEUROSCI.1774-14.2014

Demyanenko, G. P., Riday, T. T., Tran, T. S., Dalal, J., Darnell, E. P., Brennaman, L. H., et al. (2011). NrCAM deletion causes topographic mistargeting of thalamocortical axons to the visual cortex and disrupts visual acuity. J. Neurosci. 31, 1545-1558. doi: 10.1523/JNEUROSCI.4467-10.2011

Dong, H. W., Petrovich, G. D., and Swanson, L. W. (2001). Topography of projections from amygdala to bed nuclei of the stria terminalis. Brain Res. Brain Res. Rev. 38, 192-246. doi: 10.1016/S0165-0173(01)00079-0

Falk, J., Bechara, A., Fiore, R., Nawabi, H., Zhou, H., Hoyo-Becerra, C., et al. (2005). Dual functional activity of semaphorin $3 \mathrm{~B}$ is required for positioning the anterior commissure. Neuron 48, 63-75. doi: 10.1016/j.neuron.2005.10.024

Huang, H. S., Burns, A. J., Nonneman, R. J., Baker, L. K., Riddick, N. V., Nikolova, V. D., et al. (2013). Behavioral deficits in an angelman syndrome model: effects of genetic background and age. Behav. Brain Res. 243, 79-90. doi: 10.1016/j.bbr. 2012.12.052

Hull, L., Mandy, W., and Petrides, K. V. (2017). Behavioural and cognitive sex/gender differences in autism spectrum condition and typically developing males and females. Autism 21, 706-727. doi: 10.1177/1362361316669087

Imai, T., Yamazaki, T., Kobayakawa, R., Kobayakawa, K., Abe, T., Suzuki, M., et al. (2009). Pre-target axon sorting establishes the neural map topography. Science 325, 585-590. doi: 10.1126/science. 1173596

John, Y. J., Bullock, D., Zikopoulos, B., and Barbas, H. (2013). Anatomy and computational modeling of networks underlying cognitive-emotional interaction. Front. Hum. Neurosci. 7:101. doi: 10.3389/fnhum.2013.00101

Kim, S. Y., Adhikari, A., Lee, S. Y., Marshel, J. H., Kim, C. K., Mallory, C. S., et al. (2013). Diverging neural pathways assemble a behavioural state from separable features in anxiety. Nature 496, 219-223. doi: 10.1038/nature12018

Knobloch, H. S., Charlet, A., Hoffmann, L. C., Eliava, M., Khrulev, S., Cetin, A. H., et al. (2012). Evoked axonal oxytocin release in the central amygdala attenuates fear response. Neuron 73, 553-566. doi: 10.1016/j.neuron.2011.11.030

Lebow, M. A., and Chen, A. (2016). Overshadowed by the amygdala: the bed nucleus of the stria terminalis emerges as key to psychiatric disorders. Mol. Psychiatry 21, 450-463. doi: 10.1038/mp.2016.1

Leshchyns'ka, I., and Sytnyk, V. (2016). Reciprocal interactions between cell adhesion molecules of the immunoglobulin superfamily and the cytoskeleton in neurons. Front. Cell Dev. Biol. 4:9. doi: 10.3389/fcell.2016.00009

Matsuda, I., Shoji, H., Yamasaki, N., Miyakawa, T., and Aiba, A. (2016). Comprehensive behavioral phenotyping of a new Semaphorin $3 \mathrm{~F}$ mutant mouse. Mol. Brain 9:15. doi: 10.1186/s13041-016-0196-4

Matzel, L. D., Babiarz, J., Townsend, D. A., Grossman, H. C., and Grumet, M. (2008). Neuronal cell adhesion molecule deletion induces a cognitive and behavioral phenotype reflective of impulsivity. Genes. Brain Behav. 7, 470-480. doi: 10.1111/j.1601-183X.2007.00382.X

Mohan, V., Sullivan, C. S., Guo, J., Wade, S. D., Majumder, S., Agarwal, A., et al. (2018). Temporal regulation of dendritic spines through NrCAMSemaphorin3F receptor signaling in developing cortical pyramidal neurons. Cereb. Cortex doi: 10.1093/cercor/bhy004 [Epub ahead of print].

Morelli, K. H., Seburn, K. L., Schroeder, D. G., Spaulding, E. L., Dionne, L. A., Cox, G. A., et al. (2017). Severity of demyelinating and axonal neuropathy mouse models is modified by genes affecting structure and function of peripheral nodes. Cell Rep. 18, 3178-3191. doi: 10.1016/j.celrep.2017.03.009

Moy, S. S., Nadler, J. J., Young, N. B., Nonneman, R. J., Grossman, A. W., Murphy, D. L., et al. (2009a). Social approach in genetically engineered mouse lines relevant to autism. Genes Brain Behav. 8, 129-142. doi: 10.1111/j.1601-183X. 2008.00452.x

Moy, S. S., Nonneman, R. J., Young, N. B., Demyanenko, G. P., and Maness, P. F. (2009b). Impaired sociability and cognitive function in Nrcam-null mice. Behav. Brain Res. 205, 123-131. doi: 10.1016/j.bbr.2009.06.021

Paxinos, G., Halliday, G., Watson, C., Koutcherov, Y., and Wang, H. (2007). Atlas of the Developing Mouse Brain. Amsterdam: Elsevier.

Pinto, D., Pagnamenta, A. T., Klei, L., Anney, R., Merico, D., Regan, R., et al. (2010). Functional impact of global rare copy number variation in autism spectrum disorders. Nature 466, 368-372. doi: 10.1038/nature09146

Sahay, A., Molliver, M. E., Ginty, D. D., and Kolodkin, A. L. (2003). Semaphorin $3 \mathrm{~F}$ is critical for development of limbic system circuitry and is required in neurons for selective CNS axon guidance events. J. Neurosci. 23, 6671-6680. doi: 10.1523/JNEUROSCI.23-17-06671.2003

Sakurai, T. (2012). The role of NrCAM in neural development and disordersbeyond a simple glue in the brain. Mol. Cell Neurosci. 49, 351-363. doi: 10.1016/ j.mcn.2011.12.002

Schlatter, M. C., Buhusi, M., Wright, A. G., and Maness, P. F. (2008). CHL1 promotes Sema3A-induced growth cone collapse and neurite elaboration through a motif required for recruitment of ERM proteins to the plasma membrane. J. Neurochem. 104, 731-744.

Stamatakis, A. M., Sparta, D. R., Jennings, J. H., Mcelligott, Z. A., Decot, H., and Stuber, G. D. (2014). Amygdala and bed nucleus of the stria terminalis circuitry: implications for addiction-related behaviors. Neuropharmacology 76(Pt B), 320-328. doi: 10.1016/j.neuropharm.2013.05.046

Sytnyk, V., Leshchyns'ka, I., and Schachner, M. (2017). Neural cell adhesion molecules of the immunoglobulin superfamily regulate synapse formation, maintenance, and function. Trends Neurosci. 40, 295-308. doi: 10.1016/j.tins. 2017.03.003

Voineagu, I., Wang, X., Johnston, P., Lowe, J. K., Tian, Y., Horvath, S., et al. (2011). Transcriptomic analysis of autistic brain reveals convergent molecular pathology. Nature 474, 380-384. doi: 10.1038/nature10110

Xu, H. Y., Liu, Y. J., Xu, M. Y., Zhang, Y. H., Zhang, J. X., and Wu, Y. J. (2012). Inactivation of the bed nucleus of the stria terminalis suppresses the innate fear responses of rats induced by the odor of cat urine. Neuroscience 221, 21-27. doi: 10.1016/j.neuroscience.2012.06.056

Zhu, H., Pleil, K. E., Urban, D. J., Moy, S. S., Kash, T. L., and Roth, B. L. (2014). Chemogenetic inactivation of ventral hippocampal glutamatergic neurons disrupts consolidation of contextual fear memory. Neuropsychopharmacology 39, 1880-1892. doi: 10.1038/npp.2014.35

Conflict of Interest Statement: The authors declare that the research was conducted in the absence of any commercial or financial relationships that could be construed as a potential conflict of interest.

Copyright (C) 2019 Mohan, Gomez and Maness. This is an open-access article distributed under the terms of the Creative Commons Attribution License (CC BY). The use, distribution or reproduction in other forums is permitted, provided the original author(s) and the copyright owner(s) are credited and that the original publication in this journal is cited, in accordance with accepted academic practice. No use, distribution or reproduction is permitted which does not comply with these terms. 